\title{
Cost-effectiveness of adult spinal deformity surgery in a military healthcare system
}

\author{
Chris J. Neal, MD, ${ }^{1}$ Kara Mandell, PhD, ${ }^{2}$ Ellen Tasikas, MPH, ${ }^{1}$ John J. Delaney, MD, ${ }^{1}$ \\ Charles A. Miller, MD, ${ }^{1}$ Cody D. Schlaff, BS, ${ }^{3}$ and Michael K. Rosner, MD ${ }^{4}$
}

${ }^{1}$ Section of Neurosurgery, Walter Reed National Military Medical Center, Bethesda, Maryland; ${ }^{2} \mathrm{CNA}$ Corporation, Arlington, Virginia; ${ }^{3}$ George Washington University School of Medicine, Washington, DC; and ${ }^{4}$ Department of Neurosurgery, George Washington University, Washington, DC

OBJECTIVE Adult spinal deformity surgery is an effective way of treating pain and disability, but little research has been done to evaluate the costs associated with changes in health outcome measures. This study determined the change in quality-adjusted life years (QALYs) and the cost per QALY in patients undergoing spinal deformity surgery in the unique environment of a military healthcare system (MHS).

METHODS Patients were enrolled between 2011 and 2017. Patients were eligible to participate if they were undergoing a thoracolumbar spinal fusion spanning more than 6 levels to treat an underlying deformity. Patients completed the 36-Item Short Form Health Survey (SF-36) prior to surgery and 6 and 12 months after surgery. The authors used paired t-tests to compare SF-36 Physical Component Summary (PCS) scores between baseline and postsurgery. To estimate the cost per QALY of complex spine surgery in this population, the authors extended the change in health-related quality of life (HRQOL) between baseline and follow-up over 5 years. Data on the cost of surgery were obtained from the MHS and include all facility and physician costs.

RESULTS HRQOL and surgical data were available for 49 of 91 eligible patients. Thirty-one patients met additional criteria allowing for cost-effectiveness analysis. Over 12 months, patients demonstrated significant improvement $(p<$ 0.01 ) in SF-36 PCS scores. A majority of patients met the minimum clinically important difference (MCID; 83.7\%) and substantive clinical benefit threshold (SCBT; 83.7\%). The average change in QALY was an increase of 0.08. Extended across 5 years, including the $3.5 \%$ discounting per year, study participants increased their QALYs by 0.39 , resulting in an average cost per QALY of $\$ 181,649$.20. Nineteen percent of patients met the $<\$ 100,000 / Q A L Y$ threshold with half of the patients meeting the $<\$ 100,000 / Q A L Y$ mark by 10 years. A sensitivity analysis showed that patients who scored below 60 on their preoperative SF-36 PCS had an average increase in QALYs of 0.10 per year or 0.47 over 5 years.

CONCLUSIONS With a 5-year extended analysis, patients who receive spinal deformity surgery in the MHS increased their QALYs by 0.39 , with $19 \%$ of patients meeting the $\$ 100,000 /$ QALY threshold. The majority of patients met the threshold for MCID and SCBT at 1 year postoperatively. Consideration of preoperative functional status (SF-36 PCS score < 60) may be an important factor in determining which patients benefit the most from spinal deformity surgery. https://thejns.org/doi/abs/10.3171/2018.9.FOCUS18381

KEYWORDS cost-effectiveness; spinal deformity surgery; quality-adjusted life year; QALY; military healthcare system

$\mathrm{T}$ HE Institute of Medicine defines quality in health care as the "degree to which health care services for individuals and populations increase the likelihood of desired health outcomes and are consistent with current professional knowledge" and includes 6 aims: safe, effective, patient-centered, timely, efficient, and equitable. ${ }^{11}$ As medicine strives to achieve quality care, however, the question of cost becomes a significant issue. To better understand the cost of quality care, healthcare economists utilize health-related quality of life (HRQOL) measurements such as the Oswestry Disability Index, the EQ-5D, and the 36-Item Short Form Health Survey (SF-36) to calculate a

ABBREVIATIONS CEA = cost-effectiveness acceptability; GDP = gross domestic product; $\mathrm{HRQOL}=$ health-related quality of life; $\mathrm{MCID}=$ minimum clinically important difference; MHS = military healthcare system; PCS = Physical Component Summary; QALY = quality-adjusted life year; SCBT = substantive clinical benefit threshold; SF-36 = 36-Item Short Form Health Survey.

SUBMITTED August 1, 2018. ACCEPTED September 10, 2018.

INCLUDE WHEN CITING DOI: 10.3171/2018.9.FOCUS18381. 
quality-of-life valuation. Otherwise referred to as a health utility score, this value ranges from 0 to 1 and is based on particular treatments or lack thereof. The duration for a particular health utility score from a procedure or other treatment produces a quality-adjusted life year (QALY). It is important to remember that over time, QALYs are cumulative, and it is the durability of a treatment that makes it cost-effective.

The ratio of cost that is required per QALY provides the basis for cost-effectiveness is currently judged to be $\$ 100,000 /$ QALY $^{2,8,9,18}$ An intervention is considered costeffective when the cost/QALY is less than $\$ 100,000$. Some have argued, though, that inflation needs to be taken into account. This is where the concept that 3 times the gross domestic product (GDP) per capita can be used as a threshold. ${ }^{21}$ Based on the 2010 GDP per capita of the United States, this translates into \$140,580/QALY, increasing to $\$ 154,458$ based on 2015 data. ${ }^{4,8,12,15}$ Per the World Bank website, current GDP per capita in the United States is $\$ 57,638$, equating to a cost-effectiveness threshold of $\$ 172,914$ (https://data.worldbank.org/indicator/NY.GDP. PCAP.CD?view=map).

Given the controversy in the lay press over indications and outcomes associated with spine surgery, this field represents a prime area for cost-effectiveness studies. Several such studies have evaluated procedures such as anterior cervical discectomy and single-level lumbar fusions, showing them to have a cost per QALY of less than $\$ 100,000$ and, therefore, deemed cost-effective. ${ }^{1,7}$ As our population increases in age, we will continue to see more patients with adult spinal deformities. Unfortunately, the literature is lacking in quality studies that evaluate the cost-effectiveness of treatment in this patient population. As fiscal restraints become more of an issue, we must be able to determine that an intervention improves the quality of life of a patient and is cost-effective or more so than nonsurgical management. Determining whether adult spinal deformity surgery meets cost-effectiveness measures has been more elusive. With its longer operative times, increased duration of hospitalization, and known complication rates, spinal deformity surgery faces challenges to meet cost-effectiveness standards. However, alternative nonoperative management of these patients has shown little to no significant improvement in their quality of life. ${ }^{5}$

The goal of this study was to evaluate the impact that adult spinal deformity surgery has on quality of life and the cost-effectiveness of the procedure within the military healthcare system (MHS).

\section{Methods}

Prior to initiating the study, approval from the Walter Reed National Military Medical Center institutional review board was obtained. The study was an observational, prospective study with a retrospective arm dating back to 2011 until the prospective arm was initiated in 2015. There was no randomization involved and no research intervention. Individuals in the retrospective arm were approached to participate in the prospective portion of the study when they returned for standard follow-up care; only if they consented to participate were their previously collected data included for this research. If they did not return to the clinic (lost to follow-up), their retrospective information was included for the retrospective analysis only. Patients included in the study were those undergoing spinal deformity surgery who had any of following: surgery lasting $>$ 6 hours, plan to surgically treat $>6$ spinal levels, a staged procedure, significant medical comorbidities, or age $>65$ years. The cases of all complex spine patients were presented in a multidisciplinary conference in which each patient's intra- and postoperative surgical, medical, and pain care plan data were discussed and optimized.

Cost data were obtained through the MHS administrative records on all healthcare services the patient accessed during the study period, including inpatient, outpatient, and pharmacy costs. Costs were standardized to 2018 US dollars using the Bureau of Labor Statistics' Consumer Price Index-Medical Care. Costs were associated with the episode of care if they occurred within 30 days of the complex spine surgery.

Validated health outcome measures (SF-36) were then utilized pre- and postoperatively at regular intervals to determine the clinical effectiveness of the operative intervention for their disease process. Patients were included in the study if they completed an SF-36 at baseline and at follow-up visits between 1 and 36 months after surgery.

Meaningful clinical measures were identified based on the literature. We tested whether patients met the minimum clinically important difference (MCID) and the substantive clinical benefit threshold (SCBT) to identify whether the patient's health changed significantly after surgery. Copay al. found that the MCID for lumbar spine surgery was an SF-36 Physical Component Summary (PCS) score of 4.9, while Glassman et al. identified the SCBT to be a PCS score of 6.2.,3,6

Following the approach of Kharroubi et al., SF-36 scores were converted into QALYs. ${ }^{10}$ Because previous studies have found that outcomes are stable after spinal deformity surgery, we projected QALYs over 5 years and discounted them at a standard rate of $3.5 \%$ per year. To estimate the incremental cost-effectiveness ratio, baseline QALYs were extended over the same period, and the costeffectiveness acceptability (CEA) curve was estimated using nonparametric bootstrap methods.

Because previous studies have found that spinal deformity surgery provides the most benefit to the sickest patients, we conducted a sensitivity analysis focusing on patients whose baseline SF-36 PCS score was below 60.

\section{Results}

A total of 94 patients met the inclusion criteria for the study (Fig. 1). Of these, one patient declined to participate, and there were two deaths in the retrospective arm not related to the surgery, leaving 91 patients to be included in the analysis. Of the 91 eligible patients, 52 were strictly in the retrospective arm, 17 were initially retrospective and crossed over to the prospective arm, and 22 were strictly in the prospective arm. Fifty-six of the 91 patients were male $(61.5 \%)$, and the average age was 63 years old at the time of surgery (range $25-84$ years).

Forty-two patients were excluded from the outcome 


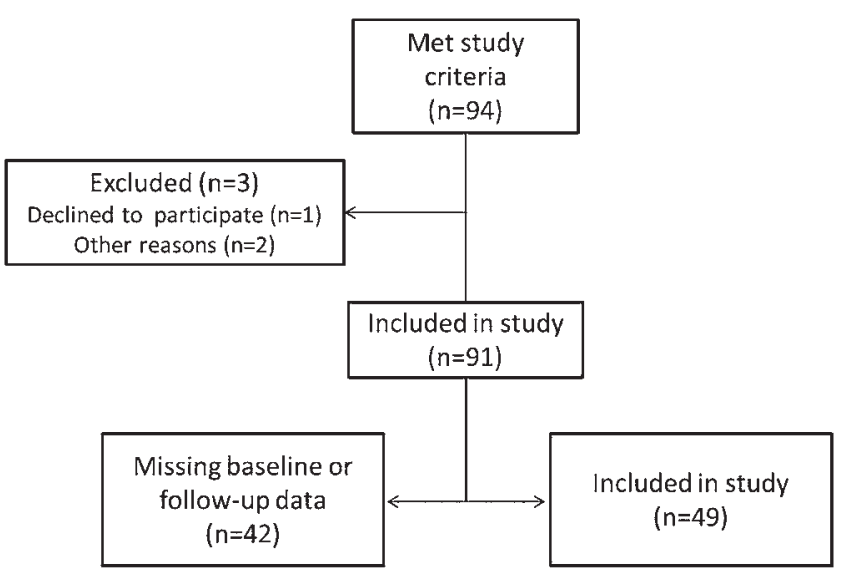

FIG. 1. Flowchart of the study inclusion process.

component of the study for lack of either baseline or appropriate follow-up HRQOL data. This left a total of 49 patients to be included in the MCID/SCBT component of the study. Of these 49 patients, $33(67.3 \%)$ were retired from the military, 14 (28.6\%) were dependents, and 2 (4.1\%) were on active duty. Both of the active duty patients underwent an L2-iliac procedure and have remained on active duty more than 1 year since surgery. Neither is undergoing medical separation from the military at this time. An additional 18 patients were excluded from the cost-effectiveness analysis due to incomplete baseline SF-36 data that prevented the calculation of QALYs. The average duration of follow-up for our cohort was 1 year. The most common type of surgery was placement of a T10-iliac construct $(79.6 \%$ in the MCID/SCBT analysis and $84.4 \%$ in the CEA analysis (Table 1).

Of the 49 patients who had complete and incomplete baseline SF-36 data with follow-up, 83.7\% met the threshold for both the MCID and the SCBT (Table 2). We observed a threshold effect such that patients who had higher SF-36 PCS scores at baseline had a smaller increase in HRQOL after spinal deformity surgery (Fig. 2).

The average cost for all healthcare services that patients accessed as part of their surgical episode of care, including inpatient, outpatient, and pharmacy costs, was $\$ 82,730.90$. The largest proportion of the cost was attributable to the cost of surgery and its associated inpatient hospital stay, which averaged \$77,472.96.

Of the 31 patients who were included in the cost-effectiveness analysis, the average change in QALY was an increase of 0.08 over a 1-year period. Extended across 5 years, including the $3.5 \%$ discounting per year, study participants increased their QALYs by 0.39 over the 5 years. This resulted in a cost per QALY of $\$ 181,649.20$. Nineteen percent of patients met the $<\$ 100,000 /$ QALY threshold. Figure 3 shows that a majority of patients exceeded the $\$ 100,000 /$ QALY threshold (64.5\%), while $16.1 \%$ experienced a decline in quality of life following surgery.

We conducted two sensitivity analyses. In the first, we limited our CEA analysis to patients whose baseline PCS score was less than 60. Among these patients, the average increase in QALYs was 0.10 per year or 0.47 over 5 years. An analysis extending our results over time (Fig. 4)
TABLE 1. Demographics of patients involved in the MCID/SCBT and the CEA analysis

\begin{tabular}{lcc}
\hline \multicolumn{1}{c}{ Variable } & $\begin{array}{c}\text { Included in } \\
\text { MCID/SCBT } \\
\text { Analysis }(\mathrm{n}=49)\end{array}$ & $\begin{array}{c}\text { Included in } \\
\text { CEA }\end{array}$ \\
\begin{tabular}{lcc} 
Analysis $(\mathrm{n}=31)$ \\
\hline male
\end{tabular} & 59.2 & 61.3 \\
\hline Average age at index surgery, yrs & 63.9 & 64.0 \\
\hline$\%$ received T10-iliac surgery & 79.6 & 87.1 \\
\hline $\begin{array}{l}\text { \% receiving other type of defor- } \\
\text { mity surgery }\end{array}$ & 20.4 & 12.9 \\
\hline $\begin{array}{l}\text { Median follow-up, yrs } \\
\text { Mean PCS score at baseline } \\
\text { (IQR) }\end{array}$ & $34.0(23.1-43.1)$ & $32.6(23.1-40.0)$ \\
\hline $\begin{array}{l}\text { \% needing revision surgery dur- } \\
\text { ing study }\end{array}$ & 6.1 & 6.5 \\
\hline
\end{tabular}

found that, by 10 years after surgery, half of participants included in the CEA analysis will meet the $<\$ 100,000 /$ QALY threshold.

\section{Discussion}

The MHS is one of the largest healthcare systems in the United States, providing care for 9.6 million beneficiaries, and it has the unique mission of providing medical support to military operations. In addition, the MHS manages a healthcare delivery system, military readiness, public health activities, medical education and training, and medical research/development. ${ }^{13}$ While there is a purchased care component outside of the military system, managed through TRICARE, the care provided within the military system itself provides an excellent opportunity to evaluate for cost since there is not a high reliance on Medicare reimbursement rates to determine the cost of a procedure, especially one as complex as adult spinal deformity surgery.

It is known that adult patients with spinal deformity have a worse HRQOL, increased use of pain medication, and less functionality. ${ }^{14}$ In 2006, Glassman et al. found the cost of nonsurgical treatment in adult scoliosis to range from $\$ 9704$ to $\$ 14,022$, with no significant changes in HRQOL. This translates into a poor cost-effective treatment strategy. ${ }^{5}$ The Spine Patient Outcome Research Trial (known as SPORT) has provided cost-effectiveness studies in evaluating nondeformity spinal surgeries and has found these procedures to be cost-effective. ${ }^{20}$ In 2014, Paulus et al. conducted a literature review to determine whether spinal deformity surgery had a positive impact on HRQOL and the cost-effectiveness of these procedures. ${ }^{14}$

TABLE 2. Changes in health state over time in the analysis and the changes in QALY for the CEA analysis

\begin{tabular}{ccc}
\hline Variable & Baseline & Follow-Up \\
\hline Mean PCS score $(n=49)$ & 34.0 & 54.6 \\
\hline Mean QALY $(n=31)$ & 0.55 & 0.64 \\
\hline
\end{tabular}




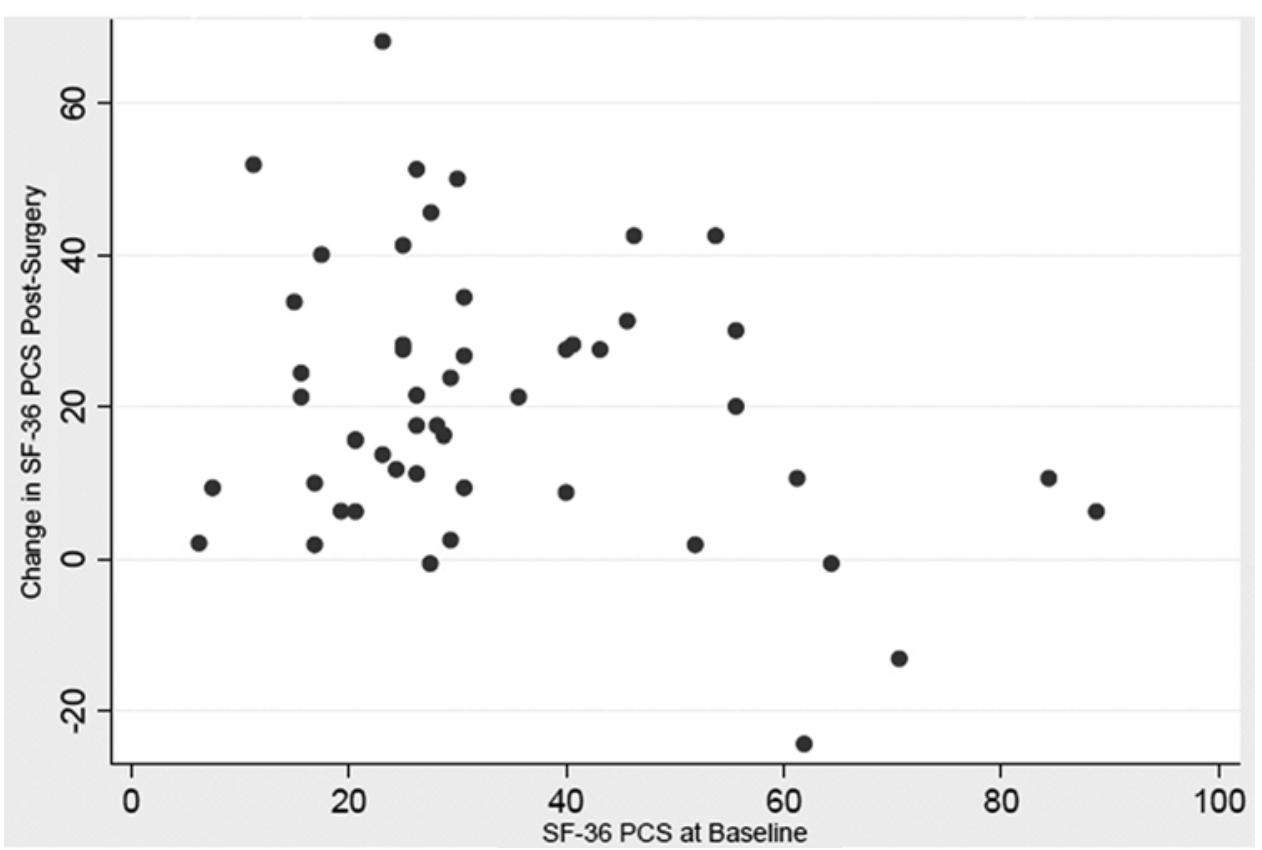

FIG. 2. Graph showing that a higher SF-36 PCS score at baseline $(>60)$ is associated with a smaller postsurgical increase.

What they found was that patients benefitted from undergoing surgery for their deformity compared to nonsurgical management, but there were sparse data in the literature regarding a cost-effectiveness analysis of the surgical and postsurgical management of these patients. Riley et al. reviewed their changes in HRQOL measurements in patients undergoing complex reconstructive spine surgery, finding that $50 \%$ of patients achieved an MCID in the Scoliosis Research Society-22R domain scores to include pain, self-image, function, and satisfaction. The authors noted that patients sustaining a neurological deficit or major complication were unlikely to achieve an MCID. ${ }^{16}$

To help determine the cost-effectiveness of spinal deformity surgery within the civilian healthcare system, Mc-

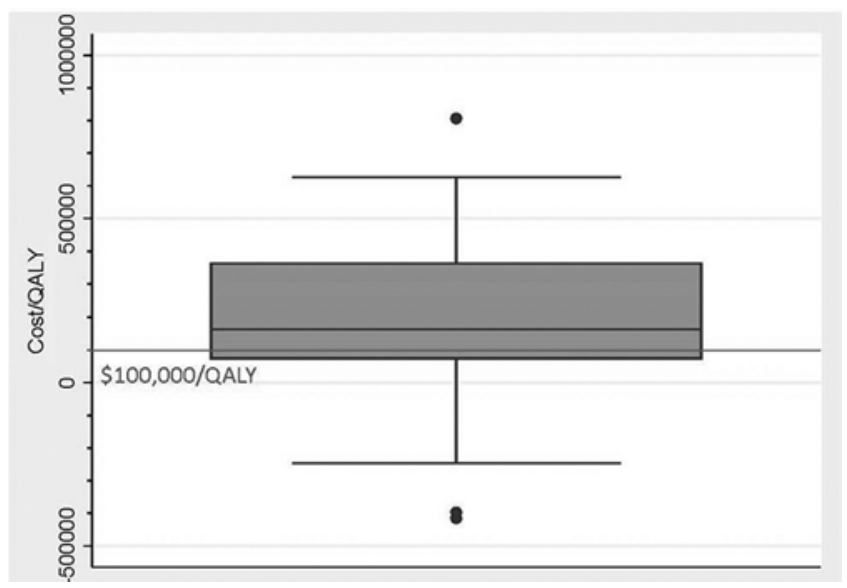

FIG. 3. Distribution of costs/QALY showing that a majority of patients (64.5\%) exceeded the $\$ 100,000 /$ Q ALY threshold, while $16 \%$ experienced a decline in quality of life following surgery.
Carthy et al. built a statistical model to predict QALYs for nonsurgical treatment and compared this with data obtained from surgical patients. Their total costs averaged $\$ 125,407$ with total QALYs of 1.93 at average 3-year follow-up. Their analysis suggests that adult spinal deformity is cost-effective after a 10 -year period. ${ }^{12}$ Terran et al. performed a multicenter study evaluating 541 patients on projected cost-effectiveness at 5 years based on a 2-year cost, determined by Medicare reimbursement rates, and HRQOL data. Less than half $(40.7 \%)$ of their patients met the cost-effectiveness threshold of $<\$ 100,000 /$ QALY with the average 5-year cost/QALY of $\$ 120,311.73 .{ }^{19}$ In a study by Fischer et al. utilizing a multicenter database that included 514 patients who underwent surgery for adult spinal deformity, the average QALY change was 0.15 and the average cost/QALY was $\$ 243,761.97$. The authors found that 56 patients (10.4\%) had a cost/QALY of less than $\$ 100,000$ at the 2-year follow-up. Age greater than 55 years, adult de novo scoliosis, prior surgery, higher preoperative sagittal vertical axis, lower maximum Cobb angles, 8 or fewer fusion levels, lower blood loss, worse global alignment classification, and global sagittal malalignment were factors that the authors found more likely to be associated with cost-effectiveness. ${ }^{4}$ The International Spine Study Group presented their research on comparing changes in QALYs after operative treatment of adult spinal deformity with nonoperative treatment. They determined that adult spinal deformity patients who underwent surgery had significantly larger increases in QALYs from baseline at 1,2, and 3 years compared with nonoperatively managed patients. The average 3-year QALY for the operative group was $0.258 \pm 0.354 . .^{17}$ Most recently, Raman et al. looked at the effect that revision surgery has on cost-effectiveness. They found that a cost/QALY at 2 years for the primary surgery was $\$ 197,809$, and it was $\$ 129,950$ for revision surgery. ${ }^{15}$ 


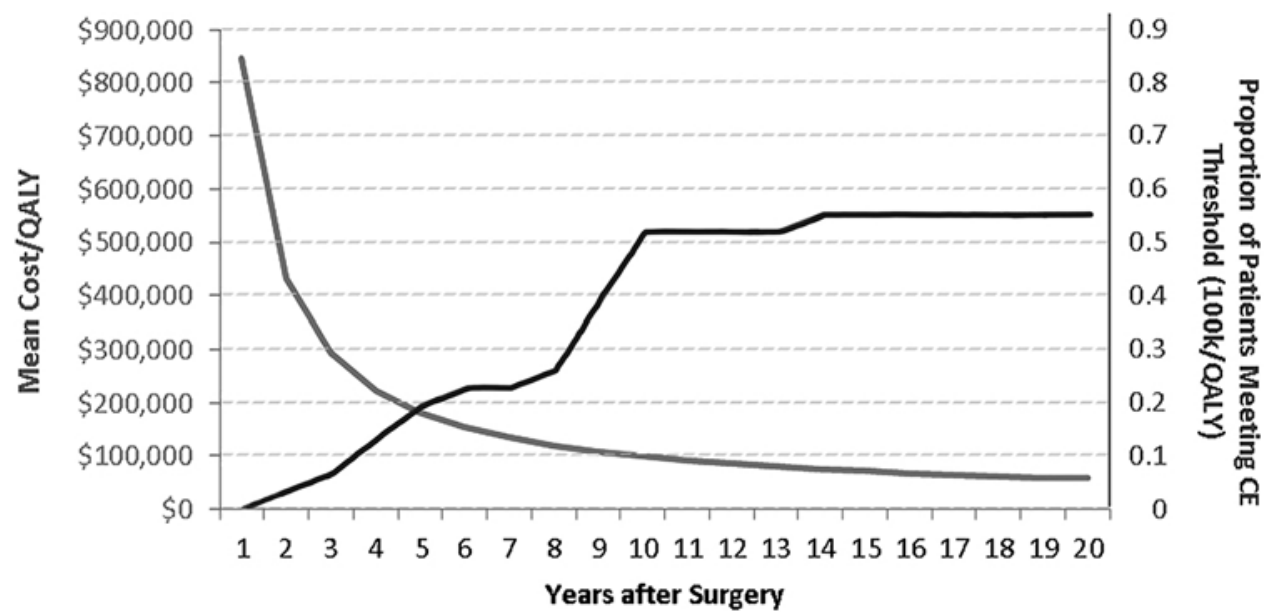

FIG. 4. Cost-effectiveness (CE) acceptability curve demonstrating that at 10 years after surgery, half of the participants included in the CEA analysis will meet the $<\$ 100,000 / Q A L Y$ threshold.

Despite the short-term follow-up, our study's findings are consistent with other data in the literature with regard to cost, especially when one takes into account the fact that those studies looked at actual costs as opposed to Medicare reimbursements. The studies that examined the actual cost from their institution for a deformity surgery found costs ranging from $\$ 115,509$ to $\$ 137,990$. . $^{12,15}$ Our cost was $\$ 82,730.90$. This compares to the studies that just used Medicare reimbursement rates, citing costs < $\$ 50,000,4,19$ When studies use two different mechanisms to evaluate costs that are more than twofold different, it makes comparing findings in the cost-effectiveness literature difficult. By looking at the actual cost to the institution, we believe that we have a truer sense of the direct costs involved. The difference in direct cost in this study compared to others can be from uncaptured costs data within the MHS as opposed to private insurance. Our QALY data of 0.08/year of follow-up is also consistent with the literature, which showed a range of 2-year cumulative QALYs to be between 0.15 and 0.4 and 3-year cumulative QALYs to be between 0.238 and 1.93 , noting that most studies find the second postoperative year typically results in a larger QALY gain than the first. ${ }^{4,12,15,17,19}$

It is interesting to note the change in QALYs (increase by 0.02 ) achieved when patients with a higher level of preoperative function (SF-36 PCS score > 60) are excluded in the analysis. This suggests that patients who have worse preoperative functional levels benefit the most from this type of surgery. Fischer et al. noted similar findings in their study in which patients with a higher level of preoperative disability were more likely to achieve the costeffectiveness threshold. ${ }^{4}$ This could represent a ceiling threshold to the treatment and may warrant consideration during the preoperative evaluation phase as to which patients would most benefit from undergoing adult spinal deformity surgery.

There are several limitations to our current study, most notably the small sample size, short follow-up duration, and the low study completion rate primarily due to the lack of baseline/follow-up HRQOL metrics in our retrospective arm. Additionally, there was no control group to compare against. The strength of this study, however, is that the cost data not were not from Medicare reimbursement rates but rather were direct costs obtained from the MHS and included all healthcare services the patient accessed during the study period, including inpatient, outpatient, and pharmacy costs.

\section{Conclusions}

Adult spinal deformity surgery performed within the MHS has outcomes and cost-effectiveness that are similar to those found in larger studies performed in civilian healthcare systems. With an average change in QALY of 0.08 per year over a 1-year period, the resulting cost per QALY with an extended 5-year analysis was \$181,649.20. Half of the patients would meet the $<\$ 100,000 /$ QALY threshold by 10 years. Consideration of preoperative functional status (SF-36 PCS score < 60) may be an important factor in determining which patients benefit the most from this type of surgery. This is the first known study to evaluate the cost-effectiveness of adult spinal deformity surgery within the MHS. Further study on this topic is required.

\section{Acknowledgments}

The authors wish to thank Surbhi Sharon Paul, MScM, for her work on obtaining cost data from the MHS administrative system.

The study received grant support from the Henry Jackson Foundation: Military Clinical Neuroscience Center of Excellence.

\section{References}

1. Carreon LY, Anderson PA, Traynelis VC, Mummaneni PV, Glassman SD: Cost-effectiveness of single-level anterior cervical discectomy and fusion five years after surgery. Spine (Phila Pa 1976) 38:471-475, 2013

2. Chapman RH, Berger M, Weinstein MC, Weeks JC, Goldie S, Neumann PJ: When does quality-adjusting life-years matter in cost-effectiveness analysis? Health Econ 13:429-436, 2004

3. Copay AG, Glassman SD, Subach BR, Berven S, Schuler TC, Carreon LY: Minimum clinically important difference in 
lumbar spine surgery patients: a choice of methods using the Oswestry Disability Index, Medical Outcomes Study questionnaire Short Form 36, and pain scales. Spine J 8:968-974, 2008

4. Fischer CR, Terran J, Lonner B, McHugh B, Warren D, Glassman S, et al: Factors predicting cost-effectiveness of adult spinal deformity surgery at 2 years. Spine Deform 2:415-422, 2014

5. Glassman SD, Berven S, Kostuik J, Dimar JR, Horton WC, Bridwell K: Nonsurgical resource utilization in adult spinal deformity. Spine (Phila Pa 1976) 31:941-947, 2006

6. Glassman SD, Copay AG, Berven SH, Polly DW, Subach BR, Carreon LY: Defining substantial clinical benefit following lumbar spine arthrodesis. J Bone Joint Surg Am 90:18391847,2008

7. Glassman SD, Polly DW, Dimar JR, Carreon LY: The cost effectiveness of single-level instrumented posterolateral lumbar fusion at 5 years after surgery. Spine (Phila Pa 1976) 37:769-774, 2012

8. Grosse SD, Teutsch SM, Haddix AC: Lessons from costeffectiveness research for United States public health policy. Annu Rev Public Health 28:365-391, 2007

9. Hirth RA, Chernew ME, Miller E, Fendrick AM, Weissert WG: Willingness to pay for a quality-adjusted life year: in search of a standard. Med Decis Making 20:332-342, 2000

10. Kharroubi SA, Brazier JE, Roberts J, O'Hagan A: Modelling SF-6D health state preference data using a nonparametric Bayesian method. J Health Econ 26:597-612, 2007

11. Lohr KN, Schroeder SA: A strategy for quality assurance in Medicare. N Engl J Med 322:707-712, 1990

12. McCarthy I, O'Brien M, Ames C, Robinson C, Errico T, Polly DW Jr, et al: Incremental cost-effectiveness of adult spinal deformity surgery: observed quality-adjusted life years with surgery compared with predicted quality-adjusted life years without surgery. Neurosurg Focus 36(5):E3, 2014

13. Military Health System: Final Report to the Secretary of Defense. Military Health System Review. (https://health. mil/Military-Health-Topics/Access-Cost-Quality-and-Safety/ MHS-Review) [Accessed October 10, 2018]

14. Paulus MC, Kalantar SB, Radcliff K: Cost and value of spinal deformity surgery. Spine (Phila Pa 1976) 39:388-393, 2014

15. Raman T, Nayar SK, Liu S, Skolasky RL, Kebaish KM: Costeffectiveness of primary and revision surgery for adult spinal deformity. Spine (Phila Pa 1976) 43:791-797, 2018

16. Riley MS, Bridwell KH, Lenke LG, Dalton J, Kelly MP: Health-related quality of life outcomes in complex adult spinal deformity surgery. J Neurosurg Spine 28:194-200, 2018

17. Scheer JK, Hostin R, Robinson C, Schwab F, Lafage V, Bur- ton DC, et al: Operative management of adult spinal deformity results in significant increases in QALYs gained compared to nonoperative management: analysis of 479 patients with minimum 2-year follow-up. Spine (Phila Pa 1976) 43:339_ 347, 2018

18. Shaw JW, Johnson JA, Coons SJ: US valuation of the EQ-5D health states: development and testing of the D1 valuation model. Med Care 43:203-220, 2005

19. Terran J, McHugh BJ, Fischer CR, Lonner B, Warren D, Glassman S, et al: Surgical treatment for adult spinal deformity: projected cost effectiveness at 5-year follow-up. Ochsner J 14:14-22, 2014

20. Tosteson AN, Tosteson TD, Lurie JD, Abdu W, Herkowitz H, Andersson G, et al: Comparative effectiveness evidence from the spine patient outcomes research trial: surgical versus nonoperative care for spinal stenosis, degenerative spondylolisthesis, and intervertebral disc herniation. Spine (Phila Pa 1976) 36:2061-2068, 2011

21. Ubel PA, Hirth RA, Chernew ME, Fendrick AM: What is the price of life and why doesn't it increase at the rate of inflation? Arch Intern Med 163:1637-1641, 2003

\section{Disclaimer}

The views expressed in this paper do not represent the official policy or opinion of the United States Navy, the United States Army, the Defense Health Agency, the Department of Defense, or the United States government.

\section{Disclosures}

Dr. Rosner reports being a consultant to Medtronic and Atec.

\section{Author Contributions}

Conception and design: Neal, Rosner. Acquisition of data: Neal, Tasikas, Delaney, Miller, Schlaff. Analysis and interpretation of data: Neal, Delaney, Schlaff. Drafting the article: Neal, Delaney, Miller, Schlaff, Rosner. Critically revising the article: Neal, Miller, Rosner. Reviewed submitted version of manuscript: Neal. Approved the final version of the manuscript on behalf of all authors: Neal. Statistical analysis: Mandell. Administrative/technical/material support: Neal, Mandell, Tasikas.

\section{Correspondence}

Chris J. Neal: Walter Reed National Military Medical Center, Bethesda, MD.christopher.j.neal3.mil@mail.mil. 